\title{
Existence of homoclinic orbits for a higher order difference system
}

\author{
Xia Liua,b,*, Tao Zhou ${ }^{\mathrm{c}}$, Haiping Shid \\ a Oriental Science and Technology College, Hunan Agricultural University, Changsha 410128, China. \\ ${ }^{b}$ Science College, Hunan Agricultural University, Changsha 410128, China. \\ ${ }^{c}$ School of Business Administration, South China University of Technology, Guangzhou 510640, China. \\ ${ }^{d}$ Modern Business and Management Department, Guangdong Construction Polytechnic, Guangzhou 510440, China. \\ Communicated by V. K. Le
}

\begin{abstract}
By using critical point theory, some new criteria are obtained for the existence of a nontrivial homoclinic orbit to a higher order difference system containing both many advances and retardations. The proof is based on the mountain pass lemma in combination with periodic approximations. Related results in the literature are generalized and improved. (C)2017 All rights reserved.
\end{abstract}

Keywords: Homoclinic orbits, higher order difference systems, critical point theory, advances and retardations. 2010 MSC: 34C37, 37J45, 39A12.

\section{Introduction}

In the theory of differential equations, the trajectories which are asymptotic to a constant state as the time variable $|t| \rightarrow \infty$ are called homoclinic orbits (or homoclinic solutions). Such orbits have been found in various models of continuous dynamical systems and frequently have tremendous effects on the dynamics of such nonlinear systems. So homoclinic orbits have been extensively studied since the time of Poincaré, see [7, 8, 17] and the references therein. Recently, Ma and Guo [14, 15] have found that the trajectories which are asymptotic to a constant state as the time variable $|k| \rightarrow \infty$ also exists in discrete dynamical systems [2-6, 11-15, 20-24, 26]. These trajectories are also called homoclinic orbits (or homoclinic solutions).

We denote by $\mathbf{N}, \mathbf{Z}$, and $\mathbf{R}$ the sets of all natural numbers, integers, and real numbers, respectively. For $a, b \in \mathbf{Z}$, define $\mathbf{Z}(a)=\{a, a+1, \cdots\}, \mathbf{Z}(a, b)=\{a, a+1, \cdots, b\}$ when $a<b$. In the following and in the sequel, for any $n \in \mathbf{N}$, we will denote the Euclidean norm in $\mathbf{R}^{\mathrm{n}}$ by $|\cdot|$, and defined as

$$
|X|=\left(\sum_{i=1}^{n} X_{i}^{2}\right)^{\frac{1}{2}}, \forall X=\left(X_{1}, X_{2}, \cdots, X_{n}\right) \in \mathbf{R}^{n} .
$$

\footnotetext{
*Corresponding author

Email addresses: xia991002@163.com (Xia Liu), zhoutaoscut@hotmail.com (Tao Zhou), shp7971@163.com (Haiping Shi)
} doi:10.22436/jnsa.010.04.44 
In this paper, we consider the following higher order nonlinear difference system

$$
\sum_{i=0}^{n} r_{i}\left(X_{k-i}+X_{k+i}\right)+x_{k} X_{k}=f\left(k, X_{k+\Gamma}, \cdots, x_{k}, \cdots, x_{k-\Gamma}\right), n \in N, k \in Z,
$$

where $r_{i}$ is real-valued for each $i \in \mathbf{Z}, \chi_{k}$ is positive real-valued for each $k \in \mathbf{Z}$, $\Gamma$ is a given nonnegative integer, $m$ is a given positive integer, $f=\left(f_{1}, f_{2}, \cdots, f_{m}\right)^{*} \in C\left(\mathbf{R}^{2 \Gamma+2} \times \mathbf{R}^{m}, \mathbf{R}\right), \chi_{k}$ and $f\left(k, Y_{\Gamma}, \cdots, Y_{0}, \cdots, Y_{-\Gamma}\right)$ are $\mathrm{T}$-periodic in $\mathrm{k}$ for a given positive integer $\mathrm{T}$.

Difference equations represent the discrete counterpart of ordinary differential equations and are usually studied in connection with numerical analysis. For the general background of difference equations, one can refer to monographs [1,19]. We may regard (1.1) as being a discrete analog of the following $2 n$ th-order differential equation

$$
\left[r(t) X^{(n)}\right]^{(n)}+\chi(t) X(t)=f(t, X(t+\Gamma), \cdots, X(t), \cdots, X(t-\Gamma)), t \in \mathbf{R} .
$$

Smets and Willem [25] had proved the existence of solitary waves with prescribed speed on infinite lattices of particles with nearest neighbor interaction for the following forward and backward differential difference equation

$$
c^{2} u^{\prime \prime}(t)=V^{\prime}(u(t+1)-u(t))-V^{\prime}(u(t)-u(t-1)), t \in R .
$$

Equations similar in structure to (1.2) arise in the study of the existence of homoclinic orbits for functional differential equations, see $[7,8]$.

When $\mathrm{m}=1, \mathrm{n}=2$ and $\Gamma=0,(1.1)$ reduces to the following special case

$$
\Delta\left(p_{k} \Delta x_{k-1}\right)-A_{k} x_{k}+b_{k} V\left(x_{k}\right)=0, k \in Z .
$$

In 2009, Deng et al. [3] applied the critical point theory to prove the existence of one homoclinic orbit for (1.3).

In 2015, Liu et al. [12] considered the existence of a nontrivial homoclinic orbit for the following equation

$$
L u_{k}-\omega u_{k}=f\left(k, u_{k+1}, u_{k}, u_{k-1}\right), k \in Z,
$$

containing both advance and retardation [27] by using the mountain pass lemma in combination with periodic approximations.

Recently, Shi et al. [22] studied the existence of a nontrivial homoclinic orbit for second order $p$ Laplacian difference equations containing both advance and retardation

$$
\Delta\left(\varphi_{p}\left(\Delta u_{k-1}\right)\right)-q_{k} \varphi_{p}\left(u_{k}\right)+f\left(k, u_{k+M}, u_{k}, u_{k-M}\right)=0, k \in \mathbf{Z},
$$

by using the critical point theory.

By establishing a proper variational framework and using the critical point theory, Chen and Tang [2] obtained some new existence criteria to guarantee the $2 \mathrm{nth}$-order nonlinear difference equation containing both many advances and retardations

$$
\Delta^{n}\left(r_{k-n} \Delta^{n} u_{k-n}\right)+q_{k} u_{k}=f\left(k, u_{k+n}, \cdots, u_{k}, \cdots, u_{k-n}\right), n \in \mathbf{Z}(3), k \in \mathbf{Z},
$$

has at least one or infinitely many homoclinic orbits.

However, to the best of our knowledge, since (1.1) contains both many advances and retardations, there are very few manuscripts dealing with this subject. The main purpose of this paper is to develop a new approach to above problem without the classical Ambrosetti-Rabinowitz condition. Motivated by the above papers [3,22], the intention of this paper is to consider problem (1.1) in a more general sense. More exactly, our results represent the extensions to a higher order nonlinear difference system containing both 
many advances and retardations. We establish some new existence criteria to guarantee that (1.1) has a nontrivial homoclinic orbit. Some existing results are generalized and improved. In fact, one can see the following Remarks 1.3 and 1.4 for details.

Throughout the paper, for a function $F$, we let $F_{i}^{\prime}\left(Y_{1}, \cdots, Y_{i} \cdots, Y_{n}\right)$ denote the partial derivative of $F$ on the $i$ variable. Let

$$
\underline{\chi}=\min _{k \in \mathbf{Z}(1, T)}\left\{\chi_{k}\right\}, \bar{\chi}=\max _{k \in \mathbf{Z}(1, T)}\left\{\chi_{k}\right\}
$$

Our main results are the following theorems.

Theorem 1.1. Assume that $\mathrm{T} \geqslant 2 \mathrm{n}+1$ and the following hypotheses are satisfied:

(r) $\mathrm{r}_{0}+\sum_{s=1}^{\mathrm{n}}\left|\mathrm{r}_{\mathrm{s}}\right|<0$;

$\left(\mathrm{F}_{1}\right)$ there exists a function $\mathrm{F}\left(\mathrm{t}, \mathrm{Y}_{\Gamma}, \cdots, \mathrm{Y}_{0}\right) \in \mathrm{C}^{1}\left(\boldsymbol{R}^{\Gamma+2} \times \boldsymbol{R}^{\mathrm{m}}, \boldsymbol{R}\right)$ such that

$$
F\left(t+T, Y_{\Gamma}, \cdots, Y_{0}\right)=F\left(t, Y_{\Gamma}, \cdots, Y_{0}\right), \sum_{i=-\Gamma}^{0} F_{2+\Gamma+i}^{\prime}\left(t+i, Y_{\Gamma+i}, \cdots, Y_{i}\right)=f\left(t, Y_{\Gamma}, \cdots, Y_{0}, \cdots, Y_{-\Gamma}\right) ;
$$

$\left(F_{2}\right)$ there exist positive constants $\rho$ and $\mathrm{a}<\frac{\underline{x}}{2(\Gamma+1)}$ such that

$$
\left|F\left(t, Y_{\Gamma}, \cdots, Y_{0}\right)\right| \leqslant a\left(\left|Y_{\Gamma}\right|^{2}+\cdots+\left|Y_{0}\right|^{2}\right)
$$

for all $\mathrm{t} \in \boldsymbol{R}$ and $\sqrt{\left|\mathrm{Y}_{\Gamma}\right|^{2}+\cdots+\left|\mathrm{Y}_{0}\right|^{2}} \leqslant \rho$;

$\left(\mathrm{F}_{3}\right)$ there exist constants $\rho, c>\frac{\bar{\chi}+\lambda_{\max }}{2(\Gamma+1)}$ and $\mathrm{b}$ such that

$$
\left|F\left(t, Y_{\Gamma}, \cdots, Y_{0}\right)\right| \geqslant c\left(\left|Y_{\Gamma}\right|^{2}+\cdots+\left|Y_{0}\right|^{2}\right)+b
$$

for all $\mathrm{t} \in \boldsymbol{R}$ and $\sqrt{\left|\mathrm{Y}_{\Gamma}\right|^{2}+\cdots+\left|\mathrm{Y}_{0}\right|^{2}} \geqslant \rho$;

$\left(\mathrm{F}_{4}\right)$ for all $\left(\mathrm{t}, \mathrm{Y}_{\Gamma}, \cdots, \mathrm{Y}_{0}\right) \in \boldsymbol{R}^{\Gamma+2} \backslash\{(0, \cdots, 0)\}$,

$$
\sum_{i=-\Gamma}^{0} F_{2+\Gamma+i}^{\prime}\left(t+i, Y_{\Gamma}, \cdots, Y_{0}\right) Y_{-i}-2 F\left(t, Y_{\Gamma}, \cdots, Y_{0}\right)>0
$$

$\left(F_{5}\right) \sum_{i=-\Gamma}^{0} F_{2+\Gamma+i}^{\prime}\left(t+i, Y_{\Gamma}, \cdots, Y_{0}\right) Y_{-i}-2 F\left(t, Y_{\Gamma}, \cdots, Y_{0}\right) \rightarrow+\infty$ as $\sqrt{\left|Y_{\Gamma}\right|^{2}+\cdots+\left|Y_{0}\right|^{2}} \rightarrow+\infty$, where $\lambda_{\max }$ can be referred to (2.4).

Then (1.1) has a nontrivial homoclinic orbit.

Remark 1.2. By $\left(\mathrm{F}_{3}\right)$, it is easy to see that there exists a constant $\zeta>0$ such that

$$
\left|F\left(t, Y_{\Gamma}, \cdots, Y_{0}\right)\right| \geqslant c\left(\left|Y_{\Gamma}\right|^{2}+\cdots+\left|Y_{0}\right|^{2}\right)+b-\zeta, \quad \forall\left(t, Y_{\Gamma}, \cdots, Y_{0}\right) \in \mathbf{R}^{\Gamma+2}
$$

As a matter of fact, let

$$
\zeta=\sup \left\{\left|F\left(t, Y_{\Gamma}, \cdots, Y_{0}\right)-c\left(\left|Y_{\Gamma}\right|^{2}+\cdots+\left|Y_{0}\right|^{2}\right)-b\right|: t \in \mathbf{R}, \sqrt{\left|Y_{\Gamma}\right|^{2}+\cdots+\left|Y_{0}\right|^{2}} \leqslant \rho\right\}
$$

we can easily get the desired result.

Remark 1.3. Theorem 1.1 extends Theorem 1.1 in [12] which is the special case of our Theorem 1.1 by letting $m=1$ and $n=2$. 
Remark 1.4. In many studies (see, e.g., $[3,4,9,11,12,14,15,23])$ of second order difference equations, the following classical Ambrosetti-Rabinowitz condition is assumed

(AR) there exists a constant $\beta>2$ such that $0<\beta F(k, u) \leqslant u f(k, u)$ for all $k \in \mathbf{Z}$ and $u \in \mathbf{R} \backslash\{0\}$.

Note that $\left(\mathrm{F}_{3}\right)-\left(\mathrm{F}_{5}\right)$ are much weaker than $(\mathrm{AR})$. Thus our result improves the existing ones.

Theorem 1.5. Assume that $\mathrm{T} \geqslant 2 \mathrm{n}+1,(\mathrm{r})$ and $\left(\mathrm{F}_{1}\right)-\left(\mathrm{F}_{5}\right)$ and the following hypothesis are satisfied:

$\left(\mathrm{F}_{6}\right) \chi_{-\mathrm{k}}=\chi_{k}, \mathrm{~F}\left(-\mathrm{k}, \mathrm{Y}_{\Gamma}, \cdots, \mathrm{Y}_{0}\right)=\mathrm{F}\left(\mathrm{k}, \mathrm{Y}_{\Gamma}, \cdots, \mathrm{Y}_{0}\right)$.

Then (1.1) has a nontrivial even homoclinic orbit.

For basic knowledge of variational methods, the reader is referred to [16, 18].

\section{Variational structure}

Our main tool is the critical point theory. We shall establish the corresponding variational framework for (1.1). We start by some basic notations for the reader's convenience.

Let $S$ be the set of sequences $X=\left(\cdots, X_{-k}, \cdots, X_{-1}, X_{0}, X_{1}, \cdots, X_{k}, \cdots\right)=\left\{X_{k}\right\}_{k=-\infty}^{+\infty}$, where $X_{k}=$ $\left(X_{k, 1}, X_{k, 2}, \cdots, X_{k, m}\right) \in \mathbf{R}^{\mathrm{m}}$.

For any $X, Y \in S, a, b \in \mathbf{R}, a X+b Y$ is defined by

$$
a X+b Y:=\left\{a X_{k}+b Y_{k}\right\}_{k=-\infty}^{+\infty} .
$$

Then $S$ is a vector space.

For any given positive integers $p$ and $T, E_{p}$ is defined as a subspace of $S$ by

$$
\mathrm{E}_{\mathrm{p}}=\left\{\mathrm{X} \in \mathrm{S} \mid \mathrm{X}_{\mathrm{k}+2 \mathrm{p} \mathrm{T}}=\mathrm{X}_{\mathrm{k}}, \forall \mathrm{k} \in \mathbf{Z}\right\} .
$$

$E_{p}$ can be equipped with the inner product $\langle X, Y\rangle$ and norm $\|X\|$ as follows,

$$
\langle X, Y\rangle:=\sum_{j=-p T}^{p T-1} X_{j} \cdot Y_{j}, \quad \forall X, Y \in E_{p}
$$

and

$$
\|X\|:=\left(\sum_{j=-p T}^{p T-1}\left|X_{j}\right|^{2}\right)^{\frac{1}{2}}, \forall X \in E_{p},
$$

where $|\cdot|$ denotes the Euclidean norm in $\mathbf{R}^{\mathrm{m}}$, and $X_{j} \cdot Y_{j}$ denotes the usual scalar product in $\mathbf{R}^{\mathrm{m}}$.

Define a linear map $M: E_{p} \rightarrow \mathbf{R}^{2 p m T}$ by

$$
M X:=\left(X_{-p T, 1}, \cdots, X_{p T-1,1}, X_{-p T, 2}, \cdots, X_{p T-1,2}, \cdots, X_{-p T, m}, \cdots, X_{p T-1, m}\right)^{*},
$$

where $X=\left\{X_{k}\right\}, X_{k}=\left(X_{k, 1}, X_{k, 2}, \cdots, X_{k, m}\right)^{*}, k \in Z(-p T, p T-1)$.

It is easy to see that the map $M$ defined in (2.1) is a linear homeomorphism with $\|X\|=|M X|$, and $\left(E_{p},\langle\cdot, \cdot\rangle\right)$ is a Hilbert space, which can be identified with $\mathbf{R}^{2 p m T}$.

For all $\mathrm{X} \in \mathrm{E}_{\mathrm{p}}$, define the functional $\mathrm{J}$ on $\mathrm{E}_{\mathrm{p}}$ as follows:

$$
J(X):=\frac{1}{2} \sum_{k=-p T}^{p T-1} \sum_{i=0}^{n} r_{i}\left(X_{k-i}+X_{k+i}\right) X_{k}+\frac{1}{2} \sum_{k=-p T}^{p T-1} X_{k}\left|X_{k}\right|^{2}-\sum_{k=-p T}^{p T-1} F\left(k, X_{k+\Gamma}, \cdots, X_{k}\right) .
$$

Since $E_{p}$ is linearly homeomorphic to $\mathbf{R}^{2 p m T}$, J can be viewed as a continuously differentiable functional defined on a finite dimensional Hilbert space. That is, $J \in C^{1}\left(E_{p}, \mathbf{R}\right)$. Furthermore, $J^{\prime}(X)=0$ if and 
only if

$$
\frac{\partial J(X)}{\partial X_{k, l}}=0, l \in Z(1, m), k \in Z(-p T, p T-1) .
$$

If we define $X_{-p T}:=X_{p T}$, then for all $l \in \mathbf{Z}(1, m), k \in \mathbf{Z}(-p T, p T-1)$,

$$
\frac{\partial J(X)}{\partial X_{k, l}}=\sum_{i=0}^{n} r_{i}\left(X_{k-i, l}+X_{k+i, l}\right)+x_{k} X_{k, l}-f_{l}\left(k, X_{k+\Gamma}, \cdots, X_{k}, \cdots, X_{k-\Gamma}\right) .
$$

Therefore, $X \in E_{p}$ is a critical point of $J$, i.e., $J^{\prime}(X)=0$ if and only if

$$
\sum_{i=0}^{n} r_{i}\left(X_{k-i, l}+X_{k+i, l}\right)+x_{k} X_{k, l}=f_{l}\left(k, X_{k+\Gamma}, \cdots, x_{k}, \cdots, X_{k-\Gamma}\right), l \in \mathbf{Z}(1, m), k \in \mathbf{Z}(-p T, p T-1) .
$$

That is,

$$
\sum_{i=0}^{n} r_{i}\left(X_{k-i}+X_{k+i}\right)+x_{k} X_{k}=f\left(k, X_{k+\Gamma}, \cdots, x_{k}, \cdots, X_{k-\Gamma}\right), k \in Z(-p T, p T-1) .
$$

On the other hand, $\left\{X_{k}\right\}_{k \in Z} \in E_{p}$ is $2 p T$-periodic in $k$ and $f\left(k, Y_{\Gamma}, \cdots, Y_{0}, \cdots, Y_{-\Gamma}\right)$ is $2 p T$-periodic in $k$. So $X \in E_{p}$ is a critical point of $J$ if and only if

$$
\sum_{i=0}^{n} r_{i}\left(X_{k-i}+X_{k+i}\right)+x_{k} X_{k}=f\left(k, X_{k+\Gamma}, \cdots, X_{k}, \cdots, X_{k-\Gamma}\right), \quad \forall k \in Z
$$

Thus, we reduce the problem of finding $2 p T$-periodic solutions of (1.1) to that of seeking critical points of the functional $J$ in $E_{p}$. For all $X \in E_{p}$, J can be rewritten as

$$
J(X)=-\frac{1}{2}\langle D M X, M X\rangle+\frac{1}{2} \sum_{k=-p T}^{p T-1} \chi_{k}\left|X_{k}\right|^{2}-\sum_{k=-p T}^{p T-1} F\left(k, X_{k+\Gamma}, \cdots, X_{k}\right),
$$

where $X=\left\{X_{k}\right\} \in E_{p}, X_{k}=\left(X_{k, 1}, X_{k, 2}, \cdots, X_{k, m}\right)^{*}, k \in Z(-p T, p T-1)$, and

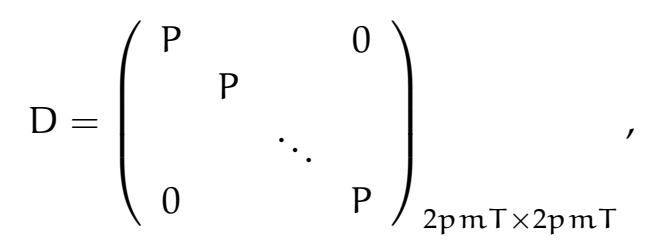

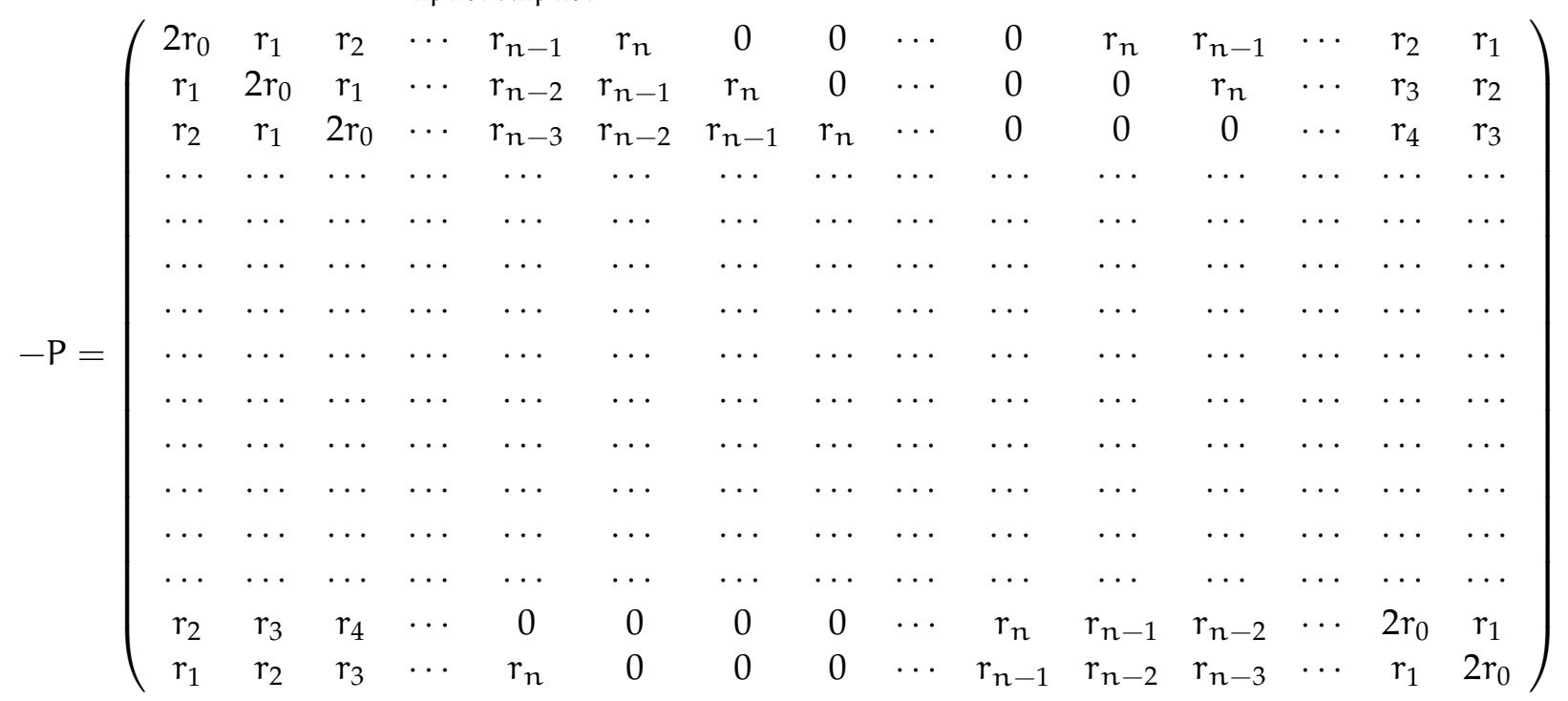


is a $2 p T \times 2 p T$ matrix. Assume that the eigenvalues of $P$ are $\lambda_{1}, \lambda_{2}, \cdots, \lambda_{T}$, and $P$ is a circulant matrix [12] denoted by

$$
P:=\operatorname{Circ}\left\{-2 r_{0},-r_{1},-r_{2}, \cdots,-r_{n}, 0, \cdots, 0,-r_{n},-r_{n-1}, \cdots,-r_{2},-r_{1}\right\} .
$$

By [10], the eigenvalues of $\mathrm{P}$ are

$$
\lambda_{j}=-2 r_{0}-\sum_{s=1}^{n} r_{s}\left\{\exp i \frac{j \pi}{p T}\right\}^{s}-\sum_{s=1}^{n} r_{s}\left\{\exp i \frac{j \pi}{p T}\right\}^{2 p T-s}=-2 \sum_{s=0}^{n} r_{s} \cos \left(\frac{j s \pi}{p T}\right),
$$

where $j=1,2, \cdots, 2 p T$. By (2.3), we know that

$$
-2 r_{0}-2 \sum_{s=1}^{n}\left|r_{s}\right| \leqslant \lambda_{j} \leqslant-2 r_{0}+2 \sum_{s=1}^{n}\left|r_{s}\right|, j=1,2, \cdots, 2 p T .
$$

It follows from (r) that $\lambda_{j}>0$ for all $j \in \mathbf{Z}(1,2 p T)$. Denote

$$
\lambda_{\max }=\max \left\{\lambda_{j} \mid \lambda_{j} \neq 0, j=1,2, \cdots, 2 p T\right\} .
$$

Let $E$ be a real Banach space, and $J \in C^{1}(E, R)$, i.e., $J$ is a continuously Fréchet-differentiable functional defined on E. J is said to satisfy the Palais-Smale condition (P.S. condition for short) if any sequence $\left\{X^{(n)}\right\}_{n \in N} \subset E$ for which $\left\{J\left(X^{(n)}\right)\right\}_{n \in N}$ is bounded and $J^{\prime}\left(X^{(n)}\right) \rightarrow 0(n \rightarrow \infty)$ possesses a convergent subsequence in $\mathrm{E}$.

\section{Main lemmas}

In order to apply critical point theory to study the existence of a nontrivial homoclinic orbit of (1.1), we shall state some lemmas, which will be used in the proofs of our main results.

Let $B_{\rho}$ denote the open ball in $E$ about 0 of radius $\rho$ and let $\partial B_{\rho}$ denote its boundary.

Lemma 3.1 (Mountain pass lemma $[16,18])$. Let $\mathrm{E}$ be a real Banach space and $\mathrm{J} \in \mathrm{C}^{1}(\mathrm{E}, \boldsymbol{R})$ satisfy the P.S. condition. If $\mathrm{J}(0)=0$ and

$\left(\mathrm{J}_{1}\right)$ there exist constants $\rho, \alpha>0$ such that $\mathrm{J}_{\partial_{\mathrm{B}}} \geqslant \alpha$, and

$\left(\mathrm{J}_{2}\right)$ there exists $\mathrm{e} \in \mathrm{E} \backslash \mathrm{B}_{\rho}$ such that $\mathrm{J}(\mathrm{e}) \leqslant 0$,

then $\mathrm{J}$ possesses a critical value $\mathrm{c} \geqslant \alpha$ given by

$$
c=\inf _{g \in \Upsilon} \max _{s \in[0,1]} J(g(s)),
$$

where

$$
\Upsilon=\{g \in C([0,1], E) \mid g(0)=0, g(1)=e\} .
$$

Lemma 3.2. Assume that $\mathrm{T} \geqslant 2 \mathrm{n}+1,(\mathrm{r})$ and $\left(\mathrm{F}_{1}\right)-\left(\mathrm{F}_{5}\right)$ are satisfied. Then $\mathrm{J}$ satisfies the P.S. condition.

Proof. Let $\left\{X^{(n)}\right\}_{n \in \mathbf{N}} \subset E_{p}$ be such that $\left\{J\left(X^{(n)}\right)\right\}_{n \in \mathbf{N}}$ is bounded and $J^{\prime}\left(X^{(n)}\right) \rightarrow 0$ as $n \rightarrow \infty$. Then there exists a positive constant $K$ such that $-K \leqslant J\left(X^{(n)}\right)$. By $\left(F_{3}^{\prime}\right)$, we have

$$
\begin{aligned}
-K \leqslant J\left(X^{(n)}\right) & \leqslant \frac{\lambda_{\max }}{2}\left\|X^{(n)}\right\|^{2}+\frac{\bar{\chi}}{2}\left\|X^{(n)}\right\|^{2}-\sum_{k=-p T}^{p T-1}\left[c\left(\left|X_{k+\Gamma}^{(n)}\right|^{2}+\cdots+\left|X_{k}^{(n)}\right|^{2}\right)+b-\zeta\right] \\
& \leqslant\left[\frac{\lambda_{\max }}{2}+\frac{\bar{\chi}}{2}-(\Gamma+1) c\right]\left\|X^{(n)}\right\|^{2}+2 p T(\zeta-b) .
\end{aligned}
$$

Therefore,

$$
\left[(\Gamma+1) c-\frac{\lambda_{\max }}{2}-\frac{\bar{X}}{2}\right]\left\|X^{(n)}\right\|^{2} \leqslant 2 p T(\zeta-b)+K .
$$

Since $c>\frac{\bar{\chi}+\lambda_{\max }}{2(\Gamma+1)},(3.3)$ implies that $\left\{X^{(n)}\right\}_{n \in N}$ is bounded in $E_{p}$. As a consequence, it has a convergent subsequence. 
Lemma 3.3. Assume that $\mathrm{T} \geqslant 2 \mathrm{n}+1,(\mathrm{r})$ and $\left(\mathrm{F}_{1}\right)-\left(\mathrm{F}_{5}\right)$ are satisfied. Then for any given positive integer $\mathrm{p},(1.1)$ possesses a $2 \mathrm{p}$ T-periodic solution $\mathrm{X}^{(\mathrm{p})} \in \mathrm{E}_{\mathrm{p}}$.

Proof. In our case, it is clear that $\mathrm{J}(0)=0$. By Lemma 3.1, J satisfies the P.S. condition. By $\left(\mathrm{F}_{2}\right)$, we have

$$
J(X) \geqslant \frac{\chi}{2}\|X\|^{2}-a \sum_{k=-p T}^{p \top-1}\left(\left|X_{k+\Gamma}\right|^{2}+\cdots+\left|X_{k}\right|^{2}\right) \geqslant \frac{X}{2}\|X\|^{2}-a(\Gamma+1)\|X\|^{2}=\left[\frac{X}{2}-a(\Gamma+1)\right]\|X\|^{2} .
$$

Taking $\alpha=\left[\frac{x}{2}-a(\Gamma+1)\right] \rho^{2}>0$, we obtain

$$
\left.\mathrm{J}(\mathrm{X})\right|_{\partial \mathrm{B}_{\rho}} \geqslant \alpha>0,
$$

which implies that $J$ satisfies the condition $\left(\mathrm{J}_{1}\right)$ of the mountain pass lemma.

Next, we shall verify the condition $\left(\mathrm{J}_{2}\right)$.

There exists a sufficiently large number $\varepsilon>\max \{\rho, \rho\}$ such that

$$
\left[(\Gamma+1) c-\frac{\lambda_{\max }}{2}-\frac{\bar{x}}{2}\right] \varepsilon^{2} \geqslant|b|
$$

Let $\vartheta \in \mathrm{E}_{\mathrm{m}}$ and

$$
\begin{gathered}
\vartheta_{k}= \begin{cases}\varepsilon, & \text { if } k=0, \\
0, & \text { if } k \in\{j \in Z:-p T \leqslant j \leqslant p T-1 \text { and } j \neq 0\},\end{cases} \\
\vdots \\
\vartheta_{k+\Gamma}= \begin{cases}\varepsilon, & \text { if } k=0, \\
0, & \text { if } k \in\{j \in Z:-p T \leqslant j \leqslant p \top-1 \text { and } j \neq 0\} .\end{cases}
\end{gathered}
$$

Then

$$
F\left(k, \vartheta_{k+\Gamma}, \cdots, \vartheta_{k}\right)= \begin{cases}F(0, \varepsilon, \cdots, \varepsilon), & \text { if } k=0, \\ 0, & \text { if } k \in\{j \in \mathbf{Z}:-p T \leqslant j \leqslant p T-1 \text { and } j \neq 0\} .\end{cases}
$$

With (3.4) and $\left(\mathrm{F}_{3}\right)$, we have

$$
\begin{aligned}
J(\vartheta) & =-\frac{1}{2}\langle\mathrm{DM} \vartheta, M \vartheta\rangle+\frac{1}{2} \sum_{k=-p T}^{p \top-1} \chi_{k}\left|\vartheta_{k}\right|^{2}-\sum_{k=-p T}^{p T-1} F\left(k, \vartheta_{k+\Gamma}, \cdots, \vartheta_{k}\right) \\
& \leqslant \frac{\lambda_{\max }}{2}\|\vartheta\|^{2}+\frac{\bar{x}}{2}\|\vartheta\|^{2}-(\Gamma+1) c\|\vartheta\|^{2}-b \\
& =-\left[(\Gamma+1) c-\frac{\lambda_{\max }}{2}-\frac{\bar{\chi}}{2}\right] \varepsilon^{2}-b \leqslant 0 .
\end{aligned}
$$

All the assumptions of the mountain pass lemma have been verified. Consequently, J possesses a critical value $c_{p}$ given by (3.1) and (3.2) with $E=E_{p}$ and $\Upsilon=\Upsilon_{m}$, where

$$
\Upsilon_{p}=\left\{g_{m} \in C\left([0,1], E_{p}\right) \mid g_{p}(0)=0, g_{p}(1)=\vartheta, \vartheta \in E_{p} \backslash B_{\varepsilon}\right\}
$$

Let $X^{(p)}$ denote the corresponding critical point of $J$ on $E_{p}$. Note that $\left\|X^{(p)}\right\| \neq 0$ since $c_{p}>0$.

Lemma 3.4. Assume that $\mathrm{T} \geqslant 2 \mathrm{n}+1,(\mathrm{r})$ and $\left(\mathrm{F}_{1}\right)-\left(\mathrm{F}_{5}\right)$ are satisfied. Then there exist positive constants $\rho$ and $\eta$ independent of $\mathrm{m}$ such that

$$
\rho \leqslant\left\|X^{(p)}\right\|_{\infty} \leqslant \eta
$$


Proof. The continuity of $\alpha \mathrm{F}\left(t, Y_{\Gamma}, \cdots, Y_{0}\right)-\sum_{i=-\Gamma}^{0} F_{2+\Gamma+i}^{\prime}\left(t, Y_{\Gamma}, \cdots, Y_{0}\right) Y_{-i}$ with respect to the variable from $Y_{\Gamma}$ to $Y_{0}$ implies that there exists a constant $\tau>0$ such that $\left|F\left(t, Y_{\Gamma}, \cdots, Y_{0}\right)\right| \leqslant \tau$ for $\sqrt{\left|Y_{\Gamma}\right|^{2}+\cdots+\left|Y_{0}\right|^{2}} \leqslant \rho$. It is clear that

$$
\begin{aligned}
J\left(X^{(p)}\right) & \leqslant \max _{0 \leqslant s \leqslant 1}\left\{-\frac{1}{2}\langle\mathrm{DM}(s \vartheta), M(s \vartheta)\rangle+\frac{1}{2} \sum_{k=-p T}^{p T-1} x_{k}\left|(s \vartheta)_{k}\right|^{2}-\sum_{k=-p T}^{p T-1} F\left(k,(s \vartheta)_{k+\Gamma}, \cdots,(s \vartheta)_{k}\right)\right\} \\
& \leqslant \frac{\left(\lambda_{\max }+\bar{\chi}\right)}{2}\|\vartheta\|^{2}+\tau=\frac{\left(\lambda_{\max }+\bar{\chi}\right)}{2} \varepsilon^{2}+\tau .
\end{aligned}
$$

Let $\xi=\frac{\left(\lambda_{\max }+\bar{\chi}\right)}{2} \varepsilon^{2}+\tau$, we have that $J\left(X^{(p)}\right) \leqslant \xi$, which is independent of $p$. From (2.2), we have

$$
\begin{aligned}
J\left(X^{(p)}\right) & =\frac{1}{2} \sum_{k=-p}^{p \top-1} \sum_{i=-\Gamma}^{0} F_{2+\Gamma+i}^{\prime}\left(k+i, X_{k+\Gamma+i}^{(p)}, \cdots, X_{k+i}^{(p)}\right) X_{k}^{(p)}-\sum_{k=-p T}^{p T-1} F\left(k, X_{k+\Gamma}^{(p)}, \cdots, X_{k}^{(p)}\right) \\
& =\frac{1}{2} \sum_{k=-p}^{p \top} \sum_{i=-\Gamma}^{0} F_{2+\Gamma+i}^{\prime}\left(k+i, X_{k+\Gamma}^{(p)}, \cdots, X_{k}^{(p)}\right) X_{k-i}^{(p)}-\sum_{k=-p T}^{p \top-1} F\left(k, X_{k+\Gamma}^{(p)}, \cdots, X_{k}^{(p)}\right) \leqslant \xi .
\end{aligned}
$$

By $\left(F_{4}\right)$ and $\left(F_{5}\right)$, there exists a constant $\eta>0$ such that for all $t \in \mathbf{R}$ and $\sqrt{\left|Y_{\Gamma}\right|^{2}+\cdots+\left|Y_{0}\right|^{2}} \geqslant \eta$,

$$
\sum_{i=-\Gamma}^{0} F_{2+\Gamma+i}^{\prime}\left(t+i, Y_{\Gamma}, \cdots, Y_{0}\right) Y_{-i}-2 F\left(t, Y_{\Gamma}, \cdots, Y_{0}\right)>\xi,
$$

which implies that $\left|X_{k}^{(p)}\right| \leqslant \eta$ for all $t \in \mathbf{R}$, that is $\left\|X^{(p)}\right\|_{\infty} \leqslant \eta$. From the definition of $J$, we have

$$
\begin{aligned}
0=\left\langle J^{\prime}\left(X^{(p)}\right), X^{(p)}\right\rangle & \geqslant \underline{x} \sum_{k=-p T}^{p T-1}\left|X_{k}^{(p)}\right|^{2}-\sum_{k=-p}^{p T} \sum_{i=-\Gamma}^{0} F_{2+\Gamma+i}^{\prime}\left(k+i, X_{k+\Gamma+i}^{(p)}, \cdots, X_{k+i}^{(p)}\right) X_{k}^{(p)} \\
& \geqslant \underline{x} \sum_{k=-p T}^{p T-1}\left|X_{k}^{(p)}\right|^{2}-\sum_{k=-p}^{p T} \sum_{i=-\Gamma}^{0} F_{2+\Gamma+i}^{\prime}\left(k+i, X_{k+\Gamma}^{(p)}, \cdots, X_{k}^{(p)}\right) X_{k-i}^{(p)} .
\end{aligned}
$$

Therefore, combined with $\left(\mathrm{F}_{2}\right)$, we get

$$
\begin{aligned}
\underline{x}\left\|X^{(p)}\right\|^{2} & \leqslant \sum_{k=-p}^{p \top} \sum_{i=-\Gamma}^{0} F_{2+\Gamma+i}^{\prime}\left(k+i, X_{k+\Gamma}^{(p)}, \cdots, X_{k}^{(p)}\right) X_{k-i}^{(p)} \\
& \leqslant \sum_{i=-\Gamma}^{0}\left[\sum_{k=-p T}^{p T-1}\left|F_{2+\Gamma+i}^{\prime}\left(k+i, X_{k+\Gamma}^{(p)}, \cdots, X_{k}^{(p)}\right)\right|^{2}\right]^{\frac{1}{2}}\left\|X^{(p)}\right\| .
\end{aligned}
$$

That is,

$$
\underline{x}\left\|X^{(p)}\right\| \leqslant \sum_{i=-\Gamma}^{0}\left[\sum_{k=-p T}^{p T-1}\left|F_{2+\Gamma+i}^{\prime}\left(k+i, X_{k+\Gamma}^{(p)}, \cdots, X_{k}^{(p)}\right)\right|^{2}\right]^{\frac{1}{2}} .
$$

Thus,

$$
\underline{x}^{2}\left\|X^{(p)}\right\|^{2} \leqslant\left\{\sum_{i=-\Gamma}^{0}\left[\sum_{k=-p \top}^{p T-1}\left|F_{2+\Gamma+i}^{\prime}\left(k+i, X_{k+\Gamma}^{(p)}, \cdots, X_{k}^{(p)}\right)\right|^{2}\right]^{\frac{1}{2}}\right\}^{2} .
$$


Combined with $\left(\mathrm{F}_{2}\right)$, we get

$$
\underline{\chi}^{2}\left\|X^{(p)}\right\|^{2} \leqslant\left\{\sum_{i=-\Gamma}^{0}\left\{\sum_{k=-p}^{p T-1}\left[2 a\left|X_{k+\Gamma+i}^{(p)}\right|\right]^{2}\right\}^{\frac{1}{2}}\right\}^{2} \leqslant 4(\Gamma+1)^{2} a^{2}\left\|x^{(p)}\right\|^{2} .
$$

Thus, we have $X^{(p)}=0$. But this contradicts $\left\|X^{(p)}\right\| \neq 0$, which shows that

$$
\left\|X^{(p)}\right\|_{\infty} \geqslant \rho,
$$

and the proof of Lemma 3.3 is finished.

\section{Proof of the main results}

Now, we shall finish out main results by using the critical point method.

Proof of Theorem 1.1. In the following, we shall give the existence of a nontrivial homoclinic orbit.

Consider the sequence $\left\{X_{k}^{(p)}\right\}_{k \in Z}$ of $2 p T$-periodic solutions found in Lemma 3.3. First, by (3.5), for any $p \in \mathbf{N}$, there exists a constant $k_{p} \in \mathbf{Z}$ independent of $p$ such that

$$
\left|X_{k_{p}}^{(p)}\right| \geqslant \rho .
$$

Since $\chi_{k}$ and $f\left(k, Y_{\Gamma}, \cdots, Y_{0}, \cdots, Y_{-\Gamma}\right)$ are all T-periodic in $k,\left\{X_{k+j}^{(p)}\right\}(\forall j \in \mathbf{N})$ is also 2pT-periodic solution of (1.1). Hence, making such shifts, we can assume that $k_{p} \in \mathbf{Z}(0, T-1)$ in (4.1). Moreover, passing to a subsequence of $p s$, we can even assume that $k_{p}=k_{0}$ is independent of $p$.

Next, we extract a subsequence, still denote by $X^{(p)}$, such that

$$
\mathrm{X}_{\mathrm{k}}^{(\mathrm{p})} \rightarrow \mathrm{X}_{\mathrm{k}}, \mathrm{p} \rightarrow \infty, \forall \mathrm{k} \in \mathbf{Z} .
$$

Inequality (4.1) implies that $\left|X_{k_{0}}\right| \geqslant \xi$ and, hence, $X=\left\{X_{k}\right\}$ is a nonzero sequence. Moreover,

$$
\begin{aligned}
& \sum_{i=0}^{n} r_{i}\left(X_{k-i}+X_{k+i}\right)+x_{k} X_{k}-f\left(k, X_{k+\Gamma}, \cdots, X_{k}, \cdots, X_{k-\Gamma}\right) \\
& \quad=\lim _{n \rightarrow \infty}\left[\sum_{i=0}^{n} r_{i}\left(X_{k-i}^{(\mathfrak{p})}+X_{k+i}^{(\mathfrak{p})}\right)+x_{k} X_{k}^{(p)}-f\left(k, X_{k+\Gamma}^{(p)}, \cdots, X_{k}^{(p)}, \ldots, X_{k-\Gamma}^{(p)}\right)\right]=0 .
\end{aligned}
$$

So $X=\left\{X_{k}\right\}$ is a solution of (1.1). Finally, for $X_{p} \in E_{p}$, let

$$
\begin{aligned}
& R_{p}=\left\{k \in \mathbf{Z}:\left|X_{k}^{(p)}\right|<\frac{\sqrt{\Gamma+1}}{\Gamma+1} \rho,-p T \leqslant k \leqslant p T-1\right\}, \\
& S_{p}=\left\{k \in \mathbf{Z}:\left|X_{k}^{(p)}\right| \geqslant \frac{\sqrt{\Gamma+1}}{\Gamma+1} \rho,-p T \leqslant k \leqslant p T-1\right\} .
\end{aligned}
$$

Since $F\left(t, Y_{\Gamma}, \cdots, Y_{0}\right) \in C^{1}\left(\mathbf{R}^{\Gamma+2} \times \mathbf{R}^{m}, \mathbf{R}\right)$, there exist constants $\bar{\xi}>0, \underline{\xi}>0$ such that

$$
\begin{aligned}
& \max \left\{\sum_{i=-\Gamma}^{0} F_{2+\Gamma+i}^{\prime}\left(k+i, Y_{\Gamma}, \cdots, Y_{0}\right): \rho \leqslant \sqrt{\left|Y_{\Gamma}\right|^{2}+\cdots+\left|Y_{0}\right|^{2}} \leqslant \eta, k \in \mathbf{Z}\right\}^{2} \leqslant \bar{\xi}, \\
& \min \left\{\sum_{i=-\Gamma}^{0} F_{2+\Gamma+i}^{\prime}\left(k+i, Y_{\Gamma}, \cdots, Y_{0}\right) Y_{-i}-F\left(k, Y_{\Gamma}, \cdots, Y_{0}\right): \rho \leqslant \sqrt{\left|Y_{\Gamma}\right|^{2}+\cdots+\left|Y_{0}\right|^{2}} \leqslant \eta, k \in Z\right\} \geqslant \underline{\xi} .
\end{aligned}
$$


For $k \in R_{p}$,

$$
\begin{aligned}
& {\left[\sum_{i=-\Gamma}^{0} F_{2+\Gamma+i}^{\prime}\left(k+i, X_{k+\Gamma}^{(p)}, \cdots, X_{k}^{(p)}\right)\right]^{2}} \\
& \quad \leqslant \frac{\bar{\xi}}{\underline{\xi}}\left\{\sum_{i=-\Gamma}^{0} F_{2+\Gamma+i}^{\prime}\left(k+i, X_{k+\Gamma}^{(p)}, \cdots, X_{k}^{(p)}\right) X_{k-i}^{(p)}-F\left(k, X_{k+\Gamma}^{(p)}, \cdots, X_{k}^{(p)}\right)\right\} .
\end{aligned}
$$

By (3.6), we have

$$
\begin{aligned}
\underline{x}^{2}\left\|X^{(p)}\right\|^{2} \leqslant & \left\{\sum_{i=-\Gamma}^{0}\left[\sum_{k \in R_{m}}\left|F_{2+\Gamma+i}^{\prime}\left(k+i, X_{k+\Gamma}^{(p)}, \cdots, X_{k}^{(p)}\right)\right|^{2}\right]^{\frac{1}{2}}\right\}^{2} \\
& +\left\{\sum_{i=-\Gamma}^{0}\left[\sum_{k \in Q_{m}}\left|F_{2+\Gamma+i}^{\prime}\left(k+i, X_{k+\Gamma^{(p)}}^{(p)}, X_{k}^{(p)}\right)\right|^{2}\right]^{\frac{1}{2}}\right\}^{2} \\
\leqslant & \left\{\sum_{i=-\Gamma}^{0}\left\{\sum_{k \in R_{m}}\left[2 a \mid X_{k+\Gamma+i}^{(p)}\right]^{2}\right\}^{\frac{1}{2}}\right\}^{2} \\
& +\frac{\bar{\xi}}{\xi}\left\{\sum_{i=-\Gamma}^{0} F_{2+\Gamma+i}^{\prime}\left(k+i, X_{k+\Gamma^{\prime}}^{(p)} \cdots, X_{k}^{(p)}\right) X_{k-i}^{(p)}-F\left(k, X_{k+\Gamma}^{(p)}, \cdots, X_{k}^{(p)}\right)\right\} \\
\leqslant & 4(\Gamma+1)^{2} a^{2}\left\|X^{(p)}\right\|^{2}+\frac{\bar{\xi} \xi}{\underline{\xi}} .
\end{aligned}
$$

Thus,

$$
\left\|x^{(p)}\right\|^{2} \leqslant \frac{\bar{\xi} \xi}{\underline{\xi}\left[\underline{\chi}^{2}-4(\Gamma+1)^{2} a^{2}\right]} .
$$

For any fixed $\mathrm{D} \in \mathbf{Z}$ and $\mathrm{p}$ large enough, we have that

$$
\sum_{k=-D}^{D}\left|x_{k}^{(p)}\right|^{2} \leqslant\left\|X^{(p)}\right\|^{2} \leqslant \frac{\bar{\xi} \xi}{\underline{\xi}\left[\underline{\chi}^{2}-4(\Gamma+1)^{2} a^{2}\right]} .
$$

Since $\bar{\xi}, \underline{\xi}, \xi, \underline{x}$ and a are constants independent of $p$, passing to the limit, we have that

$$
\sum_{k=-D}^{D}\left|X_{k}\right|^{2} \leqslant \frac{\bar{\xi} \xi}{\underline{\xi}\left[\underline{\chi}^{2}-4(\Gamma+1)^{2} a^{2}\right]}
$$

Due to the arbitrariness of $D, X$ satisfies $X_{k} \rightarrow 0$ as $|k| \rightarrow \infty$. The existence of a nontrivial homoclinic orbit is obtained.

Proof of Theorem 1.5. Consider the following boundary problem:

$$
\begin{cases}\sum_{i=0}^{n} r_{i}\left(X_{k-i}+X_{k+i}\right)+x_{k} X_{k}=f\left(k, X_{k+\Gamma}, \cdots, x_{k}, \cdots, X_{k-\Gamma}\right), & k \in \mathbf{Z}(-p T, p T), \\ \gamma_{-p T}=\gamma_{p T}=0, x_{-p T}=\chi_{p T}=0, \quad \gamma_{-k}=\gamma_{k}, x_{-k}=\chi_{k}, & k \in \mathbf{Z}(-p T, p T) .\end{cases}
$$


Let $S$ be the set of sequences $X=\left(\cdots, X_{-k}, \cdots, X_{-1}, X_{0}, X_{1}, \cdots, X_{k}, \cdots\right)=\left\{X_{k}\right\}_{k=-\infty}^{+\infty}$, where $X_{k}=$ $\left(X_{k, 1}, X_{k, 2}, \cdots, X_{k, m}\right) \in \mathbf{R}^{m}$. For any $X, Y \in S, a, b \in \mathbf{R}, a X+b Y$ is defined by

$$
a X+b Y:=\left\{a X_{k}+b Y_{k}\right\}_{k=-\infty}^{+\infty} .
$$

Then $S$ is a vector space. For any given positive integers $p$ and $T, \tilde{E}_{p}$ is defined as a subspace of $S$ by

$$
\tilde{\mathrm{E}}_{\mathrm{p}}=\left\{\mathrm{X} \in \mathrm{S} \mid \mathrm{X}_{-\mathrm{k}}=\mathrm{X}_{\mathrm{k}}, \forall \mathrm{k} \in \mathbf{Z}\right\} \text {. }
$$

$\tilde{\mathrm{E}}_{\mathrm{p}}$ can be equipped with the inner product $\langle\mathrm{X}, \mathrm{Y}\rangle$ and norm $\|\mathrm{X}\|$ as follows,

$$
\langle X, Y\rangle:=\sum_{j=-p T}^{p T} X_{j} \cdot Y_{j}, \forall X, Y \in \tilde{E}_{p T},
$$

and

$$
\|X\|:=\left(\sum_{j=-p}^{p T}\left|X_{j}\right|^{2}\right)^{\frac{1}{2}}, \forall X \in \tilde{E}_{p T},
$$

where $|\cdot|$ denotes the Euclidean norm in $\mathbf{R}^{m}$, and $X_{j} \cdot Y_{j}$ denotes the usual scalar product in $\mathbf{R}^{\mathrm{m}}$. It is obvious that $\tilde{E}_{p}$ is linearly homeomorphic to $\mathbf{R}^{2 p m T+1}$.

The techniques of the proof of Theorem 1.5 are just the same as those carried out in the proof of Theorem 1.1. For simplicity, we do not repeat them here.

\section{Acknowledgment}

This project is supported by the National Natural Science Foundation of China (No. 11401121) and Hunan Provincial Natural Science Foundation of China (No. 2015JJ2075).

\section{References}

[1] Z. Al-Sharawi (Ed.), J. M. Cushing (Ed.), S. Elaydi (Ed.), Theory and applications of difference equations and discrete dynamical systems, Proceedings of the International Conference on Difference Equations and Applications (ICDEA 2013) held at Sultan Qaboos University, Muscat, May 26-30, Springer Proceedings in Mathematics \& Statistics, Springer, Heidelberg, (2014). 1

[2] P. Chen, X.-H. Tang, Existence of homoclinic orbits for 2 nth-order nonlinear difference equations containing both many advances and retardations, J. Math. Anal. Appl., 381 (2011), 485-505. 1, 1

[3] X.-Q. Deng, G. Cheng, H.-P. Shi, Subharmonic solutions and homoclinic orbits of second order discrete Hamiltonian systems with potential changing sign, Comput. Math. Appl., 58 (2009), 1198-1206. 1, 1, 1.4

[4] X.-Q. Deng, X. Liu, H.-P. Shi, T. Zhou, Homoclinic orbits for second order nonlinear p-Laplacian difference equations, translated from Izv. Nats. Akad. Nauk Armenii Mat., 46 (2011), 17-28, J. Contemp. Math. Anal., 46 (2011), $172-182$. 1.4

[5] X.-Q. Deng, X. Liu, Y.-B. Zhang, H.-P. Shi, Periodic and subharmonic solutions for a 2 nth-order difference equation involving p-Laplacian, Indag. Math. (N.S.), 24 (2013), 613-625.

[6] X.-Q. Deng, H.-P. Shi, X.-L. Xie, Periodic solutions of second order discrete Hamiltonian systems with potential indefinite in sign, Appl. Math. Comput., 218 (2011), 148-156. 1

[7] C.-J. Guo, R. P. Agarwal, C.-J. Wang, D. O’Regan, The existence of homoclinic orbits for a class of first-order superquadratic Hamiltonian systems, Mem. Differ. Equ. Math. Phys., 61 (2014), 83-102. 1, 1

[8] C.-J. Guo, D. O'Regan, C.-J. Wang, R. P. Agarwal, Existence of homoclinic orbits of superquadratic second-order Hamiltonian systems, Z. Anal. Anwend., 34 (2015), 27-41. 1, 1

[9] Z.-M. Guo, J.-S. Yu, The existence of periodic and subharmonic solutions of subquadratic second order difference equations, J. London Math. Soc., 68 (2003), 419-430. 1.4

[10] M.-Y. Jiang, Y. Wang, Solvability of the resonant 1-dimensional periodic p-Laplacian equations, J. Math. Anal. Appl., 370 (2010), 107-131. 2

[11] X. Liu, Y.-B. Zhang, H.-P. Shi, Homoclinic orbits and subharmonics for second order p-Laplacian difference equations, J. Appl. Math. Comput., 43 (2013), 467-478. 1, 1.4 
[12] X. Liu, Y.-B. Zhang, H.-P. Shi, Homoclinic orbits of second order nonlinear functional difference equations with Jacobi operators, Indag. Math. (N.S.), 26 (2015), 75-87. 1, 1.3, 1.4, 2

[13] X. Liu, Y.-B. Zhang, H.-P. Shi, X.-Q. Deng, Periodic and subharmonic solutions for fourth-order nonlinear difference equations, Appl. Math. Comput., 236 (2014), 613-620.

[14] M.-J. Ma, Z.-M. Guo, Homoclinic orbits for second order self-adjoint difference equations, J. Math. Anal. Appl., 323 (2006), 513-521. 1, 1.4

[15] M.-J. Ma, Z.-M. Guo, Homoclinic orbits and subharmonics for nonlinear second order difference equations, Nonlinear Anal., 67 (2007), 1737-1745. 1, 1.4

[16] J. Mawhin, M. Willem, Critical point theory and Hamiltonian systems, Applied Mathematical Sciences, SpringerVerlag, New York, (1989). 1, 3.1

[17] H. Poincaré, Les méthodes nouvelles de la mécanique céleste, Gauthier-Villars, Paris, (1899). 1

[18] P. H. Rabinowitz, Minimax methods in critical point theory with applications to differential equations, CBMS Regional Conference Series in Mathematics, Published for the Conference Board of the Mathematical Sciences, Washington, DC; by the American Mathematical Society, Providence, RI, (1986). 1, 3.1

[19] H. Sedaghat, Nonlinear difference equations: Theory with applications to social science models, Springer Science \& Business Media, New York, (2013). 1

[20] H.-P. Shi, X. Liu, Y.-B. Zhang, Homoclinic orbits of second-order nonlinear difference equations, Electron. J. Differential Equations, 2015 (2015), 16 pages. 1

[21] H.-P. Shi, X. Liu, Y.-B. Zhang, Homoclinic orbits for a class of nonlinear difference equations, Azerb. J. Math., 6 (2016), 87-102.

[22] H.-P. Shi, X. Liu, Y.-B. Zhang, Homoclinic orbits for second order p-Laplacian difference equations containing both advance and retardation, Rev. R. Acad. Cienc. Exactas Fís. Nat. Ser. A Math. RACSAM, 110 (2016), 65-78. 1

[23] H.-P. Shi, H.-Q. Zhang, Existence of gap solitons in periodic discrete nonlinear Schrödinger equations, J. Math. Anal. Appl., 361 (2010), 411-419. 1.4

[24] H.-P. Shi, Y.-B. Zhang, Existence of breathers for discrete nonlinear Schrödinger equations, Appl. Math. Lett., 50 (2015), 111-118. 1

[25] D. Smets, M. Willem, Solitary waves with prescribed speed on infinite lattices, J. Funct. Anal., 149 (1997), 266-275. 1

[26] L.-W. Yang, Existence of homoclinic orbits for fourth-order p-Laplacian difference equations, Indag. Math. (N.S.), 27 (2016), 879-892. 1

[27] L.-W. Yang, Y.-B. Zhang, S.-L. Yuan, H.-P. Shi, Existence theorems of periodic solutions for second-order difference equations containing both advance and retardation, Izv. Nats. Akad. Nauk Armenii Mat., 51 (2016), 71-84. 1 